\title{
¿ES POSIBLE UNA COMPRENSIÓN ONTOLÓGICA DEL MODO DE VIDA CAMPESINA? A PROPÓSITO DE LA INVESTIGACIÓN SOBRE LOS MODOS DE EXISTENCIA DE BRUNO LATOUR
}

\section{IS AN ONTOLOGICAL UNDERSTANDING OF THE PEASANT WAY OF LIFE possible? AbOUt Bruno Latour's RESEARCH on mOdes of EXISTENCE}

Fecha recepción: 20 de julio de 2020 / fecha aceptación: 17 de marzo de 2021

América Paula Opazo Soto ${ }^{1}$

Cómo citar este artículo:

Opazo Soto, A. P. (2021). ¿Es posible una comprensión ontológica del modo de vida campesina? A propósito de la investigación sobre los modos de existencia de Bruno Latour. Revista Pensamiento y Acción Interdisciplinaria, 7(2), 87-98. https://doi.org/10.29035/pai.7.2.87

\section{Resumen}

El artículo breve que se presenta configura una reflexión sobre el modo de vida campesino y las formas ontológicas de rastrear su existencia. En ello, se plantea un recorrido conceptual sobre los enfoques vinculados a la trayectoria de la sociología del conocimiento, específicamente a la Teoría de Actor-Red y el devenir reflexivo que este ha girado hacia la comprensión onto-epistémica denominada Investigación sobre los modos de existencia (Bruno Latour). Desde lo anterior, se espera remontar, de forma reflexiva e inicial, sobre el conocimiento campesino, asociado y vinculado a infraestructuras de huertas campesinas.

Palabras clave: campesinos, ético político, modos de existencia, ontología, teoría actor-red.

1 Asistente Social, Licenciada en Trabajo Social, Magister en Ciencias Sociales y Políticas Públicas, Doctora (c) Estudios Americanos. Talca, Chile. Correo electrónico: opazo.america@gmail.com

El artículo corto que se presenta corresponde a elementos vinculados a tesis doctoral denominada "Mantenerse en la existencia campesina. Prácticas socio materiales de resistencia y cuidado en huertas de Villa Prat (Chile) y Pareditas (Argentina), postgrado realizado en el Instituto De Estudios Avanzados (IDEA), Universidad de Santiago de Chile. 


\begin{abstract}
The short article presented here is a reflection on the peasant way of life and the ontological ways of tracing its existence. In this, a conceptual tour is proposed on the approaches linked to the trajectory of the sociology of knowledge, specifically to the Actor-Network Theory and the reflexive evolution that this has turned towards the onto-epistemic understanding called Research on the modes of existence (Bruno Latour). From the above, it is expected to go back, in a reflexive and initial way, on the peasant knowledge, associated and linked to peasant orchard infrastructures.
\end{abstract}

Keywords: peasants, ethical-political, modes of existence, ontology, actor-network theory

\title{
Introducción
}

Las formas de existencia campesinas desafían al binarismo del pensamiento moderno contemporáneo que, y a modo de ejemplo, establece actores en variables dicotómicas: por un lado, un campesino viable, atento a sortear los embates del mercado agrícola, asumiendo a su vez procesos de innovación objetiva, científica y tecnológica y, por otro, un sujeto campesino al que es necesario resguardar su existencia por medio de apoyos e intervenciones estatales a fin de salvaguardar una cultura tradicional.

Apelar a la comprensión del modo de vida campesino conlleva a centrarnos en los entramados de seres de la existencia, más que humanos, conformando colectivos situados en ecologías diversas, en los que el cuidado y la resistencia se practican cotidianamente mientras se establece una infraestructura de huertas: producción de hortalizas, flores y hierbas aromáticas, configurando prácticas de intercambio y consumo alternativas al mercado convencional, en continua interrelación con ciclos de la naturaleza, en específico, los suelos.

El mantenimiento del modo de vida campesino se comprende mejor si se evita la categorización dicotómica y se adentra en aquellos seres que temporal, pero momentáneamente, enredan sus relaciones, sustentándolas. Seguir estos seres, pero manteniendo la objetividad en el proceso, es lo que apela la Investigación sobre los modos de existencia de Bruno Latour (2013); este tipo de investigación, metafóricamente hablando, podemos comprenderlo como un mapa en el que se indican ya sea miradores disponibles, puentes o refugios, etc. Así, el mapa busca que no se desvíe nuestra trayectoria; sin embargo, este mapa no asegura que realmente sigamos siempre la trayectoria indicada. Con todo, la investigación sobre los modos de existencia (AIME, por su sigla en inglés) que nos plantea Latour (2013) constituye una forma de reconocer errores ontológicos en las interrelaciones, constituyéndose en un adiestramiento para no dejar de lado el valor que la modernidad ha dado a la objetividad, pero comprendiéndola siempre mutable haciendo en el ejercicio de la investigación una serie de pruebas a fin de no desviar la búsqueda de la experiencia investigativa, mas asumiendo en ellos a actores humanos y no humanos. 


\section{Hacia una nueva visión de la ciencia: Entre los límites a la Teoría de Actor - Red y la emergencia de la Investigación sobre los Modos de Existencia}

La institucionalización de la ciencia moderna se encuentra basada en la conformación de un tipo de pensamiento binario y dual que, al igual que vectores, ha constituido su trayectoria entre un sujeto (activo) y un objeto (pasivo), siendo el criterio de la objetividad el que se ha conformado como un único modo de aprehender el conocimiento: "Cuando se apela a Ella (la Ciencia), uno ya no tiene nada que debatir puesto que se encuentra siempre en clase, en los bancos de una escuela donde la cuestión es aprender..." (Latour, 2013, p. 20); allí es donde se configura la pregunta epistemológica sobre el conocimiento.

Sin embargo, y hacia mediados de los años 70, comienza a desarrollarse la denominada sociología del conocimiento, estructurándose esta desde dos corrientes de pensamiento: el Programa Fuerte de la Escuela de Edimburgo y los Estudios de Laboratorio, las que dan paso a una serie de enfoques que en común asumen "la relativización del carácter universal y racional de la ciencia a partir de la aceptación de procesos de construcción social como constitutivos de la misma" (García Díaz, 2007, p. 173). Desde los años 80, y desde el interior de estas mismas corrientes de pensamiento, surgen críticas a los procesos de construcción social seguidos, en específico a la simetría estructural entre ciencia, tecnología y sociedad -“mientras la ciencia puede ser objeto de análisis y crítica sociológica, la sociedad se manifiesta pura e incólume" (Ureta y Sanhueza, 2018, p. 43)-, planteando la incorporación de las dimensiones materiales en la conformación del conocimiento. Desde allí, se apela a las preguntas sobre cómo lo social y el conocimiento son coproducidos y quiénes son los que participan en ese proceso.

Desde estas posturas críticas a la sociología del conocimiento, se prioriza inicialmente reconocer la Teoría de Actor-Red (TAR), liderada por los autores Bruno Latour, Michel Callon y John Law. Esta teoría establece su comienzo primero como una crítica al Programa Fuerte de la Escuela de Edimburgo, en específico a la simetría estructural que se menciona en el párrafo precedente, pero, y además, busca la independización desde los Estudios de Laboratorio, planteando el devenir de su trayectoria ya no solo por la pregunta por el conocimiento, sino que orienta su pregunta hacia lo ontológico. Desde allí, la TAR propugna que naturaleza y sociedad, en tanto actores, son siempre controversiales, pues configuran roles inesperados, inciertos o confusos. Para ello, se plantean tres principios metodológicos orientadores:

1. La peregrinación del observador se hace extensiva a las ciencias naturales y ciencias sociales. 
2. Ambas ciencias son simétricas, es decir: "los ingredientes de controversias técnicas y científicas son siempre una mezcla inseparable de consideraciones relacionadas a la sociedad y otras relacionadas a la naturaleza" (Ureta y Sanhueza, 2018, p. 44).

3. El principio de libre asociación, en donde el observador debe abandonar toda distinción a priori sobre eventos naturales y sociales, siguiendo a los actores. Este seguimiento es la estrategia para comprender cómo diversos elementos explican y construyen su mundo (Ureta y Sanhueza, 2018).

Dada la aplicación de estos tres principios, se distingue una red y la construcción de conocimientos, donde entidades sociales y naturales se configuran simultáneamente, sin distinguir con total claridad, en esas relaciones, al sujeto y objeto: quienes participan o lo que es buscado por esas interrelaciones no es controlado. Así, la TAR se constituye en un método que reúne lo semiótico y lo material, entrecruzando las relaciones entre conceptos y las cosas, conformando redes o conjunto de relaciones que configuran una realidad: "objetos, entidades, actores, procesos -todos son efectos semióticos- nodos de una red que no son más que un conjunto de relaciones" (Tirado y Doménech, 2005, p. 4).

Concentrarse en los contenidos, disputas, alianzas o negaciones al interior de las redes, más que considerar a la ciencia como un producto final, es la invitación que inicialmente realiza la TAR, pues se argumenta que las formas modernas de aprehender el conocimiento se muestran insuficientes para la comprensión de las relaciones entre naturaleza y sociedad (Latour, 2013) exhortándose a una explicación conjunta que reconozca una mayor diversidad de valores de los ya ofrecidos por la modernidad. Por tanto, la TAR reclama un estudio empírico de las prácticas científicas, apuntando a desmitificar la idea de que la ciencia moderna y su forma de producción de conocimientos responde solo a una serie de reglas metodológicas (García Díaz, 2007), girando sus preguntas hacia: ¿por qué y cómo ciertas interacciones son más exitosas, logrando estabilizar, aunque momentáneamente, una realidad?

En la trayectoria por intentar responder esta pregunta, la TAR asume al conocimiento como producto de una red de materiales heterogéneos, que yuxtapuestos, colindantes o fronterizos, van tensionando y superando sus resistencias: reconocemos que la fuerza y capacidad de organización se encuentran vinculadas a versiones teóricas asociadas al posestructuralismo francés, ya sea Guilles Deleuze, Michel Serres y Michel Foucault. De este último autor hacemos eco de que los estados de fuerzas son siempre locales e inestables (Foucault, 1976), conformando set de relaciones que, además de heterogéneas, se mantienen en tiempos y espacios situados, emergiendo de forma constante, pero momentánea, en constante proceso de cambio. 
Desde lo anterior, surge la pregunta: ¿cómo son vistos como una unidad? Es allí donde el concepto de traducción (Ureta y Sanhueza, 2018) entra en escena. Traducir es reconocer que actores humanos y no humanos "trabajan constantemente para transformar sus lenguajes, sus problemas, sus identidades o sus intereses en los de otros. A través de este proceso el mundo se construye y deconstruye, se estabiliza y se desestabiliza" (Arellano, 2003, p. 12 en Ureta y Sanhueza, 2018), haciendo, por tanto, que el orden social sea siempre emergente. Por ende, hay actores, que pueden esconder temporalmente el proceso de traducción y transformar una red heterogénea de actores y relaciones. La traducción se conforma en etapas (problematización-interesamiento-inscripciónmovilización y puntualización), las que van conformando la red: las entidades allí relacionadas, tensionadas y movilizadas cuando logran su estabilización, es decir, se conforman como una unidad, esconden la acción de los actores involucrados², conformando una caja negra, siendo por tanto la traducción la herramienta que permite seguir las redes y sus interrelaciones. En este sentido, la unidad de acción de una red puede temporalmente mantenerse dada la presencia de lo que Latour denomina móviles inmutables, es decir, las entidades se movilizan en espacio-tiempo diversos, siendo inmutables algunas de sus características que se hacen parte de su identidad, es decir, es inmutable el set de relaciones que ellas conforman. Cualquier cambio en estas entidades lo podemos denominar un cambio de paradigma.

Los cambios en las estabilizaciones de una caja negra se fundamentan en la presencia de fuerzas que resisten: las fuerzas, las entendemos desde Foucault (1976), las que son siempre locales e inestables. Desde esta perspectiva, las entidades conforman sus fuerzas desde la agencia material y social (Pickering, 1995) en tanto capacidad de actuar en la transformación del mundo. Así, las fuerzas convocan en su acción diversas resistencias y acomodaciones que, tal como tentáculos de poder, van manteniéndose o no las estabilizaciones ya mencionadas.

Ahora, la TAR y sus procesos de traducción que ellas apelan han configurado un set de críticas, inicialmente y desde los estudios feministas liderados por Donna Haraway, vinculado principalmente a la presencia de una narrativa donde predomina un lenguaje bélico y masculino (Ureta y Sanhueza, 2018): en los relatos propuestos por la TAR, estos se propugnan en el despliegue de acciones y negociaciones, donde los científicos actuarían como militares en acción.

Sumamos a lo anterior lo planteado por Pickering (1995) en cuanto a que la TAR solo alcanzaría un nivel de representación en las interacciones de humanos y

2 Reconocemos en estos postulados el interés de Bruno Latour por la episteme planteada por Etienne Souriau (2017), en su libro Los diferentes modos de existencia, donde el sujeto cognoscente y el objeto conocido no preexisten, es decir, no habría primero un pensamiento que se vuelva hacia el objeto para hacerlo existir, sino que ellos se conforman tal como una presencia indiferente, mantenidas en la opacidad. 
no humanos, en el sentido de que, al seguir los actores la descripción de las redes, puede ser interminable, no alcanzando un mayor nivel de aporte a la constitución de las prácticas científicas (Pickering, 1995). Ahondando en lo representacional de la crítica de Pickering (1995), leemos también una crítica a la idea de simetría propuesta en los principios metodológicos de la TAR. Para este autor, existe asimetría entre lo social y lo material/natural, lo que está dado en la posibilidad de intencionalidad de las acciones al interior de una práctica científica, lo que es atributo exclusivo de los actores humanos, cimentado en la configuración temporal entre ambas, basada en la capacidad de agencia ${ }^{3}$.

Autores como Decuypere y Simons (2019, en Tummons, 2020), y en forma de crítica, plantean que la TAR se constituye en una metodología socio-material esencialista de prácticas científicas, que presenta una visión problemática de la agencia no humana en el intento de aportar a, o construir, ontologías planas, pero no se observan connotaciones éticas al respecto.

Lo anterior ha ido tensionando y curvando el horizonte inicial de la TAR, donde Law (en Ureta y Sanhueza, 2018) plantea elementos de análisis que aportan a la discusión, como son la performatividad, la multiplicidad, la fluidez y política ontológica.

- La performatividad plantea que las entidades no son inmutables, sino que su trayectoria supone una continua representación de su existencia; en esa existencia representada, en la que si ocurriese algún cambio o fuera detenida, la entidad deviene en algo diferente.

- La puesta en escena de una entidad es siempre un logro momentáneo, idea que es relevada desde los aportes de Mol, quien nos dice que "las entidades son siempre más que una y menos que muchas" (Mol, 2002); es en esta puesta en escena que la autora nos plantea la idea de la traducción como un hecho que apunta a la disminución de la complejidad; esta se debe a la configuración de variados elementos, siempre múltiples y de gran complejidad y disponibles solo para algunos, temporalmente.

- La fluidez del objeto, en cuanto entidades móviles son siempre mutables, transformando las relaciones que la componen.

- Política ontológica, la que se basa en la consideración de que toda investigación realizada conlleva una responsabilidad, es decir, no solo se realiza descripción, sino que debemos hacernos cargo de esas realidades y, tal como plantea Law, "nuestros análisis y descripciones no pueden estar separados de nuestras convicciones políticas y esperanzas normativas"

3 De este modo, el fundamento de la no equivalencia entre agencia material y social es la diferencia existente en la estructura temporal de ambas, derivada de la capacidad de los agentes humanos de proyectar su acción en el tiempo a través del establecimiento de metas y el desarrollo de planes en el marco de una cultura pre-existente (Pickering, 1995, p. 176). 
(Law, 2009, p. 233 en Ureta y Sanhueza, 2018), siendo así que la práctica científica es ético-política.

Desde y en consideración de estos elementos, Bruno Latour va configurando la reflexión crítica en torno a TAR, principalmente en las interacciones de las entidades. Latour nos plantea y reconoce la robustez de esas relaciones entre diversas entidades, las cuales se desvanecen lentamente; desde allí, el autor agudiza su interés y reflexión en el encuentro entre las entidades en la red, girando aún más su reflexión desde lo epistémico hacia lo ontológico y que se manifiesta en las cosas. Para este autor, una cosa es aquello que se mantiene a través de sus manifestaciones, por tanto cuando se manifiesta ilumina lo opaco de la relación de sujeto cognoscente y objeto conocido. En este giro, Latour intenta señalar que no hay de entrada un conocimiento objetivo, sino que hay cosas cuya circulación va dando tonalidades con aquello con lo que se encuentran, siendo el conocimiento objetivo siempre mutable. Así, las cosas, y tal como un umbral, configuran un paso en la habitualidad, siempre temporal. En estas ideas se cimenta el planteamiento inicial de los modos de existencia que plantea Bruno Latour, considerándolos plurimodales, en tantos entes diferentes que coexisten y que permiten describir contemporáneamente las formas del ser, y cuyas conglomeraciones sociales, técnicas, semióticas y materiales -tales como políticas, tecnologías o moralidadesconstituyen una ontología multi- realista (Tummons, 2020).

En la base de lo anterior, reconocemos el principio de libre asociación ya mencionado en los principios metodológicos de la TAR, que, en sencillas palabras, permite a investigadores y observadores la libertad de movimiento, así como de informantes que se "asocien" y permitan dar cuenta de la continuidad del curso de acción de una realidad, situación, conformada por lo humano o no humano, y en lo cual es posible reconocer la posibilidad de mantenerse en la existencia de estos. Este mantenerse en la existencia no estaría garantizado, señala Latour, "sin pequeñas interrupciones" las que, a su vez, complejizan y extienden la red; así, al seguir el curso de una red, se dejan de lado todos los límites de la noción de dominio, pero reconociendo las diferencias entre dominios, distinguiéndose así una trayectoria. Ahora, la construcción de la trayectoria nunca es continua, sino que se ensancha o comprime, se vigoriza o se debilita, pero siempre se mantiene algo, lo que permite acceder a fenómenos lejanos y, tal como plantea Latour (2013), es como "si entre el autor y el fenómeno se haya tendido una especie de puente que ahora otros también pueden cruzar". Ese puente es lo que los investigadores Ilaman "suministrar la prueba de la existencia de un fenómeno" (Latour, 2013, p. 52). En ello, el establecimiento y rastreo de redes presenta una fecha de caducidad, pues y desde las diferencias que permite la construcción de la trayectoria no es eterna, conformando discontinuidades. Así, el autor, al centrarse en las discontinuidades, intenta enfocar su lente hacia lo onto-epistémico, intentado "captar una continuidad a través de una serie de discontinuidades, otra 
continuidad pasando por otra discontinuidad" (Latour, 2013, p. 53), por donde se traza la red. Ahora, en la red se deben reconocer los seres por los cuales hay que pasar para que una situación se mantenga, lo que se reconstituye por medio de una prueba, una investigación, una innovación o una crisis, por donde es posible pasar para devenir o seguir siendo el mismo, sin hacer nada.

Así, los modos de existencia de Latour toman los límites de la traducción que plantea su Teoría de Actor-Red, cuya vocación se refiere a que ninguna realidad se encuentre sin representación, pero ahonda en la posibilidad de comprobar la pertenencia onto-epistémica de sus trayectorias, siendo así que un modo de existencia es siempre una versión del "SER EN CUANTO OTRO" (una muestra de discontinuidad y de continuidad, de diferencia y de repetición, de lo otro y de lo mismo) y, a la vez, un régimen propio de veridicción (Latour, 2013, p. 182-183). Desde esta perspectiva, y en el supuesto que Latour asume tácitamente las críticas de ontologías planas y/o de redes poderosas y dominantes, los modos de existencia y su investigación proporcionan una forma de pensar las diferencias cualitativas entre diversos actores de la red, a fin de poner a prueba los propios procesos de objetividad.

\section{Traduciendo la metodología de la investigación sobre los modos de existencia propuesto por Bruno Latour. Un adiestramiento para alcanzar la experiencia de la institución moderna en la que se instala el campesinado}

94 Bajo el contexto recién referenciado, Bruno Latour (2013) -al proponer la Investigación sobre los modos de existencia, en tanto una antropología de los modernos- apela a configurar que el conocimiento aprehendido por medio de la institucionalidad moderna logre la instauración ${ }^{4}$ de la objetividad: en ello, el autor apuesta que la subjetividad de los investigadores se ponga a prueba por medio de una serie de pruebas $^{5}$ para alcanzar, en esta experiencia, el buen pensar objetivo de investigadoras e investigadores. Latour ha inventariado quince modos de existencia, todos ellos ordenados en cinco grupos: el ordenamiento, no azaroso por cierto, intenta descomponer nuestras configuraciones epistémicas aprendidas en la institucionalidad moderna sobre el sujeto y el objeto. Así, en cada grupo se califica la objetividad, o sea, se busca instituir confianza de y en las verdades alcanzadas, atendiendo en ello al reconocimiento de los valores propios

\footnotetext{
4 Para el autor, el conocimiento moderno en tanto institución es frágil y delicada, pues su mantenimiento es posible por el aprendizaje de método y, en ello, Latour (2013) se pregunta cómo hacer más robusta dicha institucionalidad.

5 En el dispositivo de Cuadro Cruzado que propone el autor, los modos de existencia inventariados se someten a las siguientes pruebas: HIATO y TRAYECTORIA, CONDICIONES DE FELICIDAD E INFELICIDAD, SERES POR INSTAURAR y ALTERACIONES, respectivamente. Compartimos con Souriau (2017) que las pruebas como tal son peligrosas, pues tienen como objetivo hacer vacilar las trayectorias modernas de aprehender al conocimiento.
} 
de una institución, en interacción con los valores que ya se encuentran instituidos en contrastes 6 .

Este adiestramiento metodológico pasa por la comprensión y apropiación de dispositivo de Cuadro Cruzado creado por Latour para calificar cada modo de existencia, exponiéndolos a las pruebas ya referidas, las que, y tal como pequeñas interrupciones, complejizan y extienden las interacciones en la negociación entre actores humanos y no humanos en tanto red, las que al seguir su curso van dejando tras de sí una trayectoria que traspasa todos los límites de la noción de dominio. Con todo, este ejercicio, no sencillo por lo demás, tiene la intencionalidad de, por medio de las pruebas ya mencionadas, abrir la epistemología moderna a un sendero que permita reconocer la instauración de la convergencia de modos de existencia; en ello, la investigación sobre los modos de existencia constituye una apuesta onto-epistémica posible de utilizar continuamente en cada nueva investigación empírica y filosófica (Hamalainen y Lehtonen, 2016), relevando en este ejercicio la preocupación por la consumación de la objetividad, recorriendo en ello "el largo trayecto que va desde el bosquejo hasta la obra, sin recurrir a ninguno de los modelos conocidos de realización, de construcción, de creación, de emergencia o de planificación" (Souriau, 2017, p. 12).

En la investigación de los modos de existencia se hace central comprender la noción de errores de categoría planteada por Latour (2013). De acuerdo a Tummons (2020), el error de categoría se debe sintetizar como un error ontológico, es decir, cuando algunas propiedades de una unidad que se presentan son consideradas como una propiedad diferente. Al aclarar esas interrelaciones y sus propiedades es posible construir un relato desde la resolución de los errores de categoría.

En su adiestramiento, Latour (2013) nos plantea que toda investigación parte por los modos de existencia de red [RES] y el modo de existencia de la preposición [PRE] interrelacionados: la [RES], en un símil con la teoría de Actor-Red, nos permite rastrear la red de asociaciones y conexiones de los actores humanos y no humanos, hasta donde sea necesario; por su parte, [PRE] es la que permite calificar las asociaciones y conexiones que permite la extensión de la red (Tummons, 2020); la convergencia [RES - PRE] autoriza el despliegue descriptivo de nuestras investigaciones, pues y desde ellas se podrán demostrar las distinciones de lo interrelacionado: las tensiones entre distintos actores, sus pasiones, intenciones, acercándonos a reconocer todo el color que se encuentra en acciones realizadas. Desde allí es posible rastrear y perfilar los distintos modos de existencia inventariados por Latour, poniendo a prueba la objetividad de investigadores.

6 Los contrastes suponen el adiestramiento de un formato para aprehender al conocimiento. Es con este ejercicio que se apela a la generación de confianza en los procesos intelectuales desarrollados. 


\section{Remontando sobre el conocimiento campesino en infraestructuras de huertas. Indicios de una investigación}

Ir más allá de la institucionalidad científica moderna que ha aprehendido al conocimiento campesino es lo que se ha buscado relevar en la investigación basada en la infraestructura de huertas: estas, como un entramado de hortalizas, hierbas medicinales, flores y árboles frutales, configuran un set de prácticas que cotidianamente allí son desarrollados por campesinas y campesinos. En el adiestramiento que propone Bruno Latour (2013) ponemos inicialmente sobre la mesa la concepción moderna sobre la cual el campesinado ha sido construido, dicotómicamente?.

Reconociendo estas distinciones modernas, giramos nuestro interés en rastrear todos los actores que se vinculan interna como externamente en una huerta, por medio de una indagación empírica, soportada en procesos etnográficos. Al respecto, Bernasconi (2015) plantea que los métodos etnográficos y sus características naturalista, abierta, procesual y flexible, constituyen un proceso de producción de conocimientos que permite estudiar las prácticas y en ello "cómo el individuo es hecho existir no genéricamente, sino en situación -como el producto una red fluida de relaciones- y como un objeto múltiple que gestiona diversas existencias" (Bernasconi, 2015, p.215). Así, y planteando la convergencia [RES - PRE] que inicialmente nos plantea Latour (2013), en una infraestructura de huertas campesinas están todos incluidos: campesinos, campesinas, suelos, agua, semillas, estaciones del año, sol, lluvia. De este modo, actores humanos y no humanos son los participantes que componen las huertas. Entonces, tanto material y semióticamente los actores involucrados conforman, todos, la huerta campesina.

Ahora, perfilar este tipo de investigaciones, además de apelar a la búsqueda de objetividad en los procesos investigativos, nos pone en la tensión de que las decisiones metodológicas que se tomen conlleven decisiones ético-políticas en el hacer investigación. Es en el giro de esas decisiones que reconocemos que no pueden independizarse nuestras descripciones de nuestras "convicciones políticas y esperanzas normativas" (Law, 2009, p. 233 en Ureta y Sanhueza, 2018). Por tanto, y al plantear el seguir los seres de la existencia, en específico la posibilidad de intentar una comprensión ontológica del modo de vida campesina, planteamos la necesidad de que, en el rastreo de las distintas redes, se establezca también el seguimiento del hacer ético-político en cuanto forma de aprehender al conocimiento.

\footnotetext{
7 Si retomamos algunas corrientes teóricas sobre campesinos, por ejemplo, los enfoques campesinistas/descampesinistas que apelan a elementos económicos que priorizan su existencia, principalmente a la capacidad de adaptación que estos presentan y han desarrollado; así se ha perfilado que instituciones como la Organización de las Naciones Unidas para la Alimentación y la Agricultura (FAO) denominen estos modos de vida como agricultura familiar campesina, con distintas clasificaciones de acuerdo a la disponibilidad de ingresos y de acceso a los recursos disponibles.
} 


\section{Referencias bibliográficas}

Arellano, A. (2003) La sociología de las ciencias sociales y de las técnicas de Bruno Latour y Michell Callon. Cuadernos Digitales: Publicación electrónica en Historia, Archivística y Estudios Sociales, 8(23).

Bernasconi, O. (2015). ¿Qué nos hace ser individuos? Por un enfoque posthumano, pragmático y relacional. Athenea Digital. Revista de pensamiento e investigación social, 15(2), 205-229. https://www.redalyc.org/articulo. oa? id $=53741125008$

Foucault, M. (1976). Vigilar y Castigar: el nacimiento de la prisión. Siglo XXI.

García, M. (2007). ¿Es posible una historia constructivista de la dimensión material de la ciencia? A propósito de la historiografía poshumanista de Andrew Pickering. En P. Lorenzano y H. Miguel (Comp.), Filosofía e historia de la ciencia en el cono sur (pp. 173-181). Editorial Educando.

García Díaz, P. (2007). Bruno Latour y los límites de la descripción en el estudio de la ciencia. [Tesis doctoral], Departamento de Filosofía, Universidad de Granada.

Hamalainen N. y Lehtonen, T. (2016). Latour's empirical metaphysics, Distinktion: Journal of Social Theory, 17(1), 20-37. https://doi.org/10.1080/160091 OX.2016.1154883

Latour, B (2013). Investigación sobre los modos de existencia. Una antropología de los modernos. Paidós.

Mol, A. (2002). The Body Multiple: Ontology in Medical Practice. Duke University Press.

Pickering, A. (1995). The mangle of Practice: Time, Agency, and Science. University of Chicago Press.

Souriau, E. (2017). Los diferentes modos de existencia. Editorial Cactus.

Tirado, F. y Doménech, M. (2005) Asociaciones heterogéneas y actantes: El giro postsocial de la teoría del actor red. Revista de Antropología Iberoamericana, 1-26. https://ddd.uab.cat/record/199541

Tummons, J. (2020) Higher education, theory, and modes of existence: thinking about universities with Latour, Higher Education Research \& Development, 40(6). 1-13. https://doi.org/10.1080/07294360.2020.1804337

Ureta, S. y Sanhueza, N. (2018) Emergencia de una disciplina: los estudios CTS en el mundo y Latinoamérica. En A. Espinosa-Rada, F. Ortiz y N. Sanhueza (Comps), Tecnopolíticas: aproximaciones a los estudios de ciencia, tecnología y sociedad en Chile (pp.19-66). Editorial UAH. 
¿Es posible una comprensión ontológica del modo de vida campesina? A propósito de la investigación sobre los modos de existencia de Bruno Latour por América Paula Opazo Soto

\section{Dirección de correspondencia:}

América Paula Opazo Soto

Contacto: opazo.america@gmail.com

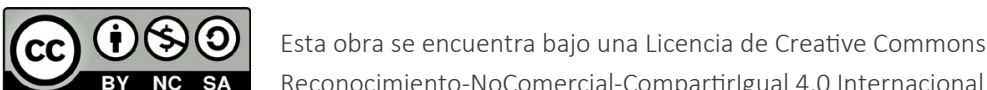

Escuela de Trabajo Social · Universidad Católica del Maule 